\title{
Designing adjuvant treatment based on biological measurements in the neoadjuvant setting
}

\author{
Ian E Smith* and Aekaterini Kotsori
}

For many years, one of the main aims of neoadjuvant therapy has been to identify short term endpoints that will predict for long-term outcome in adjuvant treatment. The advantages are obvious: in contrast to adjuvant trials, neoadjuvant trials require hundreds rather than thousands of patients, are very much less expensive to run and produce outcome data many years earlier.

A key primary endpoint for neoadjuvant trials has been objective clinical response and this still remains the case [1]. Nevertheless, major studies have frequently shown that clinical response, including complete clinical remission, does not predict for long-term outcome. For example, in the National Surgical Adjuvant Breast and Bowel Project (NSABP) B-18 trial no difference in outcome was seen between patients who achieved clinical response versus those who did not [2]. Likewise, in the NSABP B-27 trial in which 2,411 patients were randomised to neoadjuvant adriamycin/cyclophosphamide (AC) versus the same treatment followed by sequential docetaxel, the complete response rate was $64 \%$ for the sequential, docetaxel-containing arm compared with $40 \%$ for the AC arm alone $(P<0.001)$, but no significant difference was seen in long-term survival [3,4]. Likewise, for neoadjuvant endocrine therapy, the IMPACT (Immediate Preoperative Anastrozole, Tamoxifen, or Combined With Tamoxifen) trial compared neoadjuvant anastrazole versus tamoxifen versus the combination and was the neoadjuvant equivalent of the ATAC (Arimidex, Tamoxifen, Alone or in Combination) adjuvant trial. No significant difference was seen in response rate between the three arms of IMPACT [5] and this failed, therefore, to correlate with the long-term disease-free survival advantage of anastrazole in ATAC. Likewise, in the IMPACT trial a large difference was seen in response rates for patients with human epidermal growth factor receptor 2 (HER2)-positive tumours in favour of anastrazole (7 of 12 responders; 58\%) compared with

*Correspondence: ian.smith@rmh.nhs.uk

Breast Unit, The Royal Marsden Hospital and Institute of Cancer Research, Fulham Road, London, SW3 6JJ, UK tamoxifen (2 of 9 responders; 22\%) and yet no selective disease-free survival advantage for anastrazole over tamoxifen in patients with HER2-positive tumours was subsequently seen in ATAC [6]. A possible explanation for this is that it is entirely possible that tumour growth rate may be slowed by neoadjuvant therapy (as measured, for example, by a reduction in proliferation factor Ki67) without a formal clinical response being achieved but with a consequent gain in relapse free survival. In the IMPACT trial, for example, only $37 \%$ of tumours achieved an objective clinical response rate to anastrazole whereas 75\% had a significant reduction in Ki67 [7].

In contrast to clinical complete remission, pathological complete remission ( $\mathrm{pCR}$ ) (involving complete pathological disappearance of tumour from most breast and axillary nodes) following neoadjuvant chemotherapy has been shown to be of major prognostic significance in many studies $[2,8]$. Differences in PCR have also sometimes been shown to reflect differences in long-term outcome in randomised trials. For example, in patients with HER2-positive tumours, two major trials have shown that the addition of trastuzumab to neoadjuvant chemotherapy achieves a major increase in pCR rates $[9,10]$, results that accurately reflect the very significant disease-free survival and overall survival improvement achieved with the addition of trastuzumab to chemotherapy in adjuvant trials $[11,12]$.

There are, however, problems with pCR. First, not all trials have shown a correlation between $\mathrm{pCR}$ and longterm outcome. In particular, in the largest neoadjuvant chemotherapy trial, NSABP B-27, sequential treatment with docetaxel after AC achieved a $26 \%$ pCR rate compared with $13 \%$ for $\mathrm{AC}$ alone $(P<0.001)$ and yet no significant survival difference subsequently emerged between the two arms [3,4]. Second, pCR rates following neoadjuvant chemotherapy are relatively low, ranging from around 10 to 20\%; this end point applies, therefore, to only a minority of patients and misses many others who also have a good prognosis. In particular, the $\mathrm{pCR}$ rate following chemotherapy is very low in patients with oestrogen receptor-positive tumours at around 8\% [13] and yet many of these patients not achieving pCR nevertheless have an excellent prognosis. The next issue is 
that $\mathrm{pCR}$ is very rarely seen following neoadjuvant endocrine therapy and is not, therefore, a useful endpoint in this major subgroup. Finally pCR as an endpoint is uninformative until treatment has been completed and surgical excision carried out; it does not, therefore, allow for early changes in treatment.

Biological parameters predicting for pCR after chemotherapy, or better still predicting for long-term outcome irrespective of $\mathrm{pCR}$, would therefore be very valuable. One such widely studied parameter is Ki67 as a measure of proliferation. Ki67 has been shown to predict for $\mathrm{pCR}$ after chemotherapy as has oestrogen receptor status, HER2 status and grade [14]. However, in this study only HER2 was predictive in multivariant analysis. In a review, Ki67 was predictive for pCR in six out of eight studies by univariate analysis but only one out of five studies in multivariate analysis [15]. There is, however, a paradox with Ki67 since high levels, although predictive for $\mathrm{pCR}$, nevertheless are associated with significant adverse prognosis [14]. The likely explanation for this paradox is that high Ki67 predicts for a minority of patients who will achieve pCR and do well but also for a majority who will not and will do badly.

A further problem with classical neoadjuvant therapy trials is that they nearly always relate to large cancers, usually with diameters of $3 \mathrm{~cm}$ or more. The question arises as to whether outcome parameters in these accurately predict for outcome in smaller cancers associated with standard adjuvant therapy.

A possible solution to many of these problems is the use of short-term preoperative therapy for around 2 weeks before surgery. In the IMPACT trial of neoadjuvant endocrine therapy described above we were able to show in multivariate analysis that higher Ki67 expression after 2 weeks of endocrine therapy was statistically significantly associated with lower recurrence-free survival $(P=0.004)$ whereas higher Ki67 expression at baseline prior to treatment was not. These findings suggest that measurements of Ki67 and other molecular markers after short-term preoperative treatment may be more useful in predicting long-term outcome than similar parameters measured at baseline. Furthermore, the approach can be applied to all patients requiring adjuvant therapy, in contrast to the minority with large cancers eligible for classical neoadjuvant treatment, and results can be obtained quickly with the opportunity of changing therapy if required.

In conclusion, in standard neoadjuvant therapy, clinical response is an unreliable endpoint for outcome. $\mathrm{pCR}$ is a better predictor but is useful only in a minority of patients, is of no use for endocrine therapy and is too late to influence treatment. The entire classical neoadjuvant approach is based on the uncertain premise that large primaries (which are uncommon) reflect the treatment sensitivity of small primaries (which are common). Short duration (2 to 3 weeks) preoperative therapy could provide molecular endpoints reflecting both innate tumour biology and treatment effect. These could involve the majority of patients with early breast cancer and this approach overcomes many of the limitations of classical neoadjuvant studies.

\section{Abbreviations}

AC, adriamycin/cyclophosphamide; ATAC, Arimidex, Tamoxifen, Alone or in Combination; HER2, human epidermal growth factor receptor 2; IMPACT, Immediate Preoperative Anastrozole, Tamoxifen, or Combined With Tamoxifen; NSABP, National Surgical Adjuvant Breast and Bowel Project; pCR, pathological complete remission.

\section{Competing interests}

The authors declare that they have no competing interests.

\section{Acknowledgements}

This article has been published as part of Breast Cancer Research Volume 12 Supplement 4, 2010: Controversies in Breast Cancer 2010. The full contents of the supplement are available online at http://breast-cancer-research.com/ supplements/12/s4

Published: 20 December 2010

\section{References}

1. Alba E, Calvo L, Albanell J, De la Haba J, Chacon J, Arcusa Lanza A, Sanchez Roviraet $\mathrm{P}$ : Chemotherapy (CT) versus hormone therapy $(\mathrm{HT})$ as neoadjuvant treatment in luminal breast cancer: a multicenter, randomized phase II study (GEICAM/2006-03) [abstract]. J Clin Oncol 2010, 28:15s.

2. Wolmark N, Wang J, Mamounas E, Bryant J, Fisher B: Preoperative chemotherapy in patients with operable breast cancer: nine-year results from National Surgical Adjuvant Breast and Bowel Project B-18. J Natl Cancer Inst Monogr 2001, 30:96-102.

3. Bear HD, Anderson S, Brown A, Smith R, Mamounas EP, Fisher B, Margolese R, Theoret H, Soran A, Wickerman DL, Wolmark: National Surgical Adjuvant Breast and Bowel Project Protocol B-27. The effect on tumor response of adding sequential preoperative docetaxel to preoperative doxorubicin and cyclophosphamide: preliminary results from National Surgical Adjuvant Breast and Bowel Project Protocol B-27. J Clin Oncol 2003, 21:4165-4174.

4. Bear HD, Anderson S, Smith RE, Geyer CE Jr, Mamounas EP, Fisher B, Brown AM, Robidoux A, Margolese R, Kahlenberg MS, Paik S, Soran A, Wickerham DL, Wolmark N: Sequential preoperative or postoperative docetaxel added to preoperative doxorubicin plus cyclophosphamide for operable breast cancer:National Surgical Adjuvant Breast and Bowel Project Protocol B-27. J Clin Oncol 2006, 24:2019-2027.

5. Smith IE, Dowsett M, Ebbs SR, Dixon JM, Skene A, Blohmer JU, Ashley SE, Francis S, Boeddinghaus I, Walsh G, IMPACT Trialists Group; Neoadjuvant treatment of postmenopausal breast cancer with anastrozole, tamoxifen, or both in combination: the Immediate Preoperative Anastrozole, Tamoxifen, or Combined with Tamoxifen (IMPACT) multicenter doubleblind randomized trial. J Clin Oncol 2005, 23:5108-5116.

6. Dowsett M, Allred C, Knox J, Quinn E, Salter J, Wale C, Cuzick J, Houghton J, Williams N, Mallon E, Bishop H, Ellis I, Larsimont D, Sasano H, Carder P, Cussac AL, Knox F, Speirs V, Forbes J, Buzdar A: Relationship between quantitative estrogen and progesterone receptor expression and human epidermal growth factor receptor 2 (HER-2) status with recurrence in the Arimidex, Tamoxifen, Alone or in Combination trial. J Clin Oncol 2008, 26:1059-1065.

7. Dowsett M, Smith IE, Ebbs SR, Dixon JM, Skene A, Griffith C, Boeddinghaus I, Salter J, Detre S, Hills M, Ashley S, Francis S, Walsh G, IMPACT Trialists: Shortterm changes in Ki67 during neo adjuvant treatment of primary breast cancer with anastrazole or tamoxifen alone or combined correlate with recurrence free survival. Clin Cancer Res 2005, 11:951s-968s

8. Jones RL, Smith IE: Neoadjuvant treatment for early-stage breast cancer:opportunities to assess tumour response. Lancet Oncology 2006, 7:869-874 
9. Buzdar AU, Ibrahim NK, Francis D, Booser DJ, Thomas ES, Theriault RL, Pusztai L, Green MC, Arun BK, Giordano SH, Cristofanilli M, Frye DK, Smith TL, Hunt KK, Singletary SE, Sahon AA, Ewer MS, Buchholz RA, Berry D, Hortobagyi GN: Significantly higher pathologic complete remission rate after neoadjuvant therapy with trastuzumab, paclitaxel, and epirubicin chemotherapy: results of a randomized trial in human epidermal growth factor receptor 2-positive operable breast cancer. JCO 2005, 23:3676-3685.

10. Gianni L, Eiermann W, Semiglazov V, Manikhas A, Lluch A, Tjulandin S, Zambetti M, Vazquez F, Byakhow M, Lichinitser M, Climent MA, Ciruelos B, Mansutti M, Bozhok A, Baronio R, Feyereislova A, Barton C, Valagussa P, Baselga J: Neoadjuvant chemotherapy with trastuzumab followed by adjuvant trastuzumab versus neoadjuvant chemotherapy alone, in patients with HER2-positive locally advanced breast cancer (the NOAH trial): a randomised controlled superiority trial with a parallel HER2negative cohort. Lancet 2010, 375:377-384.

11. Mackey J, McLeod D, Ragaz J, Gelmon K, Verma S, Pritchard K, Laing K Provencher L, Charbonneau LF: Adjuvant targeted therapy in early breast cancer. Cancer 2009, 115:1154-1168.

12. Slamon D, Eiermann W, Robert N: Phase III randomized trial comparing doxorubicin and cyclophosphamide followed by docetaxel (ACT) with doxorubicin and cyclophosphamide followed by docetaxel and trastuzumab (ACTH) with docetaxel, carboplatin and trastuzumab (TCH) in Her2neu positive early breast cancer patients: BCIRG 006 Study [abstract]. Cancer Res 2009, 69:500s.

13. Ring AE, Smith IE, Ashley S, Fulford LG, Lakhani SR: Oestrogen receptor status, pathological complete response and prognosis in patients receiving neoadjuvant chemotherapy for early breast cancer. $\mathrm{Br} J$ Cancer 2004, 91:2012-2017.

14. Jones RL, Salter J, A'Hern R, Nerurkar A, Parton M, Reis-Filho JS, Smith IE, Dowsett M: The prognostic significance of Ki67 before and after neoadjuvant chemotherapy in breast cancer. Breast Cancer Res Treat 2009, 116:53-68.

15. Yerushalmi R, Woods R, Ravdin PM, Hayes MM, Gelmon KA: Ki67 in breast cancer: prognostic and predictive potential. Lancet Oncol 2010, 11:174-183.

doi: $10.1186 /$ bcr 2745

Cite this article as: Smith IE, Kotsori A: Designing adjuvant treatment based on biological measurements in the neoadjuvant setting. Breast Cancer Research 2010, 12(Suppl 4):S16. 